\title{
Polycystic ovary syndrome in adolescents
}

\begin{abstract}
Objective: To propose to the clinical evidence available in the literature based on the criteria, recommendations and implications in addressing adolescent patients which condition decisions that impact treatment and follow-up to adulthood.
\end{abstract}

Method: search was conducted in PubMed, Uptodate, Medical Subjetc Headings of articles published in English with the following keywords (Mesh): Polycystic ovary syndrome in adolescents, metabolic syndrome, hyperandrogenism in adolescent disorders menstrual pattern in adolescents. Inclusion criteria: systematic review and meta-analysis.

Results: 24 articles were selected, among them 10 are review articles, 11 original studies, 2 meta-analyzes and one consensus. Diagnostic criteria overlap with the physiological changes including clinical manifestations of hyperandrogenism and disorders in menstrual pattern in which mention should be made to diagnose polycystic ovary syndrome in this population, considering that the phenotype change through the reproductive life and teens have a heterogeneous ovarian morphology was proposed that the diagnosis was later confirmed the 18 years the first-line treatment includes oral contraceptives, change in lifestyle, use of insulin sensitizers to improve cardiovascular risk and alterations associated metabolic

Conclusion: Polycystic ovary syndrome in adolescents is a challenging diagnosis for the clinician, and there has been a consensus that persistent unexplained hyperandrogenic anovulation using age appropriate and stage are criteria appropriate diagnostic ovary syndrome standards polycystic adolescents, be warned by consensus not to label hyperandrogenic adolescents as polycystic ovary syndrome if the menstrual abnormality has not persisted for 2 years or more before that time, it is recommended to consider patients as at risk of syndrome polycystic ovary, early diagnosis and early treatment radically change the prevalence of comorbiditiesit should promote a holistic approach to maximize the future health of our patients.

Keywords: polycystic ovary syndrome, polycystic ovary syndrome in adolescents, hyperandrogenism in adolescents, menstrual disorders in adolescent pattern, contraception, metabolic syndrome
Volume 10 Issue 4 - 2019

\author{
Edgar Allan Villagomez mendoza,' Manuel \\ Martinez Meraz ${ }^{2}$ \\ 'Third-year resident physician of Gynecology and Obstetrics, \\ Mexico \\ ${ }^{2} \mathrm{OB}-\mathrm{GYN}$ physician, biologist and researcher human reproduction, \\ Mexico \\ Correspondence: Dr. Edgar Allan Villagomez Mendoza, Hospital \\ General Dr. Jose Maria Rodriguez, lago nyasa 46 jardines de \\ morelos, Ecatepec, Mexico, Tel 5539547359, \\ Email allan.villagomez.m@gmail.com
}

Received: August 16,2019 | Published: August 26, 2019
Abbreviations: PCOS, polycystic ovary syndrome; FSH, follicle stimulating hormone; LH, luteinizing hormone; BMI, body mass index; DHEA, dehydroepiandrosterone; IGF-1, insulin-like growth factor 1; GABA, gamma-aminobutyric acid; LHCGR, LH receptor choriogonadotropin

\section{Summary}

Ovarian hyperandrogenism associated oligoanovulacion is the most common cause of hirsutism, acne and menstrual disorders in adolescents pathophysiology of polycystic ovary syndrome (PCOS) involves starting interactions of genetic, epigenetic changes, primary ovarianabnormalities, neuroendocrine disturbances, hyperinsulinemia, insulin resistance, obesity, international companies have reached a consensus in the persistent unexplained hyperandrogenic anovulation using standards applied to their age and stage are considered suitable for polycystic ovary syndrome in adolescents diagnostic criteria. It is the most common endocrine disorder heterogeneous in women of reproductive age.

\section{Background}

The polycystic ovary syndrome was originally described by Stein and Leventhal in 1935 as the association of amenorrhea with polycystic ovaries, 5 of the 7 original cases had hirsutism and acne, 4 were obese, over the past 25 years have developed new diagnostic criteria for adults based on various combinations of hyperandrogenism and anovulation unexplained cause, all covered by the Rotterdam consensus criteria, resulting in four phenotypes Table 1. However the usefulness of these criteria result in a problem when applied to the adolescent population, so in 2015 international pediatric societies describe a consensus for diagnostic criteria suitable for SOP and adolescents, with appropriate modification to the age and stage. ${ }^{1,2}$ 
Table I Criteria polycystic ovary syndrome

\begin{tabular}{lll}
\hline Workgroup & Date & Definition \\
\hline NIH & 1990 & $\begin{array}{l}\text { Chronic anovulation, hyperandrogenism clinical or biochemical and exclusion of other } \\
\text { pathologies }\end{array}$ \\
ESHRE-ASRM/Rotterdam 2003 & $\begin{array}{l}\text { The presence of two of the three criteria: olio-anovulation, clinical or biochemical } \\
\text { hyperandrogenism and polycystic ovarian morphology }\end{array}$ \\
AES & 2009 & $\begin{array}{l}\text { Hyperandrogenism or hyperandrogenemia and ovulatory dysfunction (oligo-anovulation } \\
\text { and polycystic ovaries) and exclusion of other pathologies. }\end{array}$
\end{tabular}

\section{Epidemiology}

The polycystic ovary syndrome is the most common endocrine disease in women of reproductive age, the National Institutes of Health reports a prevalence of $6 \%$, however, it is difficult to ascertain the prevalence in adolescents because of the diversity on diagnostic criteria and the fact that many signs and symptoms of PCOS may overlap with normal puberty, consequently there have been several studies to evaluate the prevalence of PCOS in adolescents, a study in India of 778 adolescents and young women aged 15 to 24 years it was estimated a prevalence of $22.5 \%$ for Rotterdam criteria and $10.7 \%$ for criteria Androgen Excess Society, patients with PCOS nonobese corresponded to $71 \%$ of cases, in another study of Caucasian, Hispanic and African-American girls, 24.2\% showed the presence of SOP, limitations in the interpretation of these data is related in most of ethnicity and lack of prevalence studies based on specific criteria for the adolescent population. ${ }^{3,4}$

\section{Risk factor's}

Described the presence of early puberty with an incidence of $32 \%$, as has been reported alteration in the gene of androgens, increased methylation of the androgen receptor, increased factors insulinlike growth, changes in other genes involved in steroidogenesis as 21-hydroxylase, among others, so it is considered that early puberty is associated with ovarian hyperandrogenism, and as an early marker of future diseases, family history of mother with polycystic ovary syndrome contributing to a hereditary component, obesity and valproic acid as is described in the literature which increases transcription of steroidogenic CYP7 gene causing increasing serum testosterone. ${ }^{3,5}$

\section{Genetic role}

Two studies of genome-wide association in Chinese whose condition was diagnosed using the criteria of Rotterdam identified 11 genomic loci associated with regulation of steroid hormone and the insulin signaling, polymorphism of a nucleotide was located in the region of a polypeptide follicle stimulating hormone (FSH) and ARL15P genes, this nucleotide polymorphism is strongly associated with luteinizing hormone $(\mathrm{LH})$, other genes located in the $8 \mathrm{p} 23$ locus are GATA4, NEIL2 and FDFT1. Another study demonstrated complete genome in a phase followed by replication in women with this condition signals were located near these ERBB4, FSHB, RAD50 genes and KRR1 European population. ${ }^{6}$

\section{Metabolic markers in PCOS}

In one study the association of activin and follistatin as markers for SOP investigated, elevated serum levels of follistatin found in PCOS patients regardless of age and body mass index (BMI) levels activin had no impact compared to patients no SOP, it was found that follistatin inhibits FSH, is also a promoter in the inflammation process which has been shown related to insulin resistance, also other peptide hormones related to SOP is adiponectin and ghrelin, which is have negatively correlated with levels of circulating androgens which supports a relationship with the hyperandrogenic condition of SOP and its associated metabolic abnormalities, It is further described regarding leptin levels with respect to deterioration lipid profile particularly LDL and insulin resistance.,

\section{Pathophysiology}

Androgen excess, observed in about 60 to $80 \%$ of patients with PCOS, is a key feature of the disorder. Hirsutism and hyperandrogenism are manifestations of excessive androgen production. In fact, hyperandrogenism, commonly demonstrated by increased free testosterone in circulation, is the most common abnormality seen in the syndrome and plays an important role in perpetuating taxpayers abhorrent to the pathophysiology of PCOS hormones. Excessive ovarian androgen production is present in most cases, but the overproduction of adrenal androgens may occur among some.

The pathophysiological disorder in its main components described pathophysiology primary ovarian, factors influencing follicular growth are coordinated so that, in general, there is only a single follicle selected for terminal maturation and ovulation sequentially. In the SOP, the balance between androgens AMH (HAM) and FSH is altered, leading to follicular arrest. LH abundant causes the theca cells to produce androgens, the concentrations of FSH and the conversion of androgens to estradiol are insufficient, which makes it possible not select a dominant follicle, resulting in chronic anovulation, the AMH, secreted by granulosa cells, It plays an important role in managing this balance because it inhibits the transition from primary to primary follicles. Therefore, PCOS is characterized by increased growth of the small follicles, but subsequent growth arrest leads to polycystic typical morphology. The theca cells obtained from women with PCOS retain their phenotype with increased androgen secretion due to increased expression of CYP17A1 or activity P450c17, many steroidogenic enzymes are expressed in both the adrenal cortex, particularly in the reticular zone, the hormones secreted by this area include dehydroepiandrosterone (DHEA), DHEA and androstenedione sulfate. It is increasingly evident that the repertoire of the adrenal steroidogenic cells and, perhaps.

PCOS women are at increased risk of developing impaired glucose tolerance and type 2 diabetes, the pathogenesis of insulin resistance in PCOS reflects the interaction of genetic influences, intrauterine environmental factors and inheritable extrauterine, however in the context of SOP, puberty per se could play an important role in the 
molecular origins of hyperinsulinemia. During puberty, teenagers experience a temporary decrease in insulin sensitivity with a nadir at puberty, this was attributed to increases in growth hormone and concentrations of insulin-like growth factor 1 (IGF-1) this growth period to provide more amino acids.

Obesity in patients with PCOS occurs in $40-80 \%$ of cases the result is the presence of hypertrophic adipocytes which have lipolytic activity, ie, increase in free fatty acids contributes to hepatic insulin resistance, accumulation lipid, i.e. diacylglycerol and ceramides in muscle and liver interferes with insulin signaling, intracellular ceramides may also alter insulin signaling to block translocation of Akt, an important mediator of insulin sensitivity, to the plasma membrane, this also causes the increase in macrophage infiltration producing proinflammatory substances such as TNF alpha, IL-6, leptin and resistin, serine phosphorylation stimulating 1 (IRS1) inhibiting insulin signaling,this leads to a decreased glucose transporter GLUT4 receptor, in turn free fatty acids bind to Toll-like receptors initiating the cascade of proinflammatory mediators, increasing insulin resistance, the latter present in the $30-70 \%$ PCOS patients causing hyperinsulinism, increasing the pulses in the central nervous system specifically hypothalamus increasing GnRH secretion, which stimulates the pituitary to the LH surge causing cell hyperplasia teak therefore increase in androgens circulating insulin normally stimulates aromatase activity in granulosa cells for the conversion of androgens into estrogens teak, for insulin resistance phenomenon does not occur,therefore the presence of acanthosis nigricans as skin sign of insulin resistance, which is described as hyperkeratotic plaques, papillomatosis and hyperpigmentation as hyperinsulinemia increases the affinity for the insulin growth factor dela skin exerting production and proliferation fibroblasts, melanocytes and keratinocytes dermal.

Changes in the secretion of GnRH and gonadotropin in the SOP although it is not mandatory for diagnosis is the presence of dysregulated secretion of gonadotropins, LH and FSH, which control ovarian steroidogenesis, follicular dynamics and ovulation, therefore it is reasonable to assume that the secretory profiles altered gonadotropins might affect the cardinal features of SOP, including hyperandrogenism and ovulatory dysfunction there is evidence suggesting that elevated androgen disrupt capacity of sex steroids to regulate the secretion of GnRH and LH through classic feedback cycles, this would result in a decreased negative feedback actions of ovarian steroids that contribute and perpetuate hypersecretion LH feature of PCOS.
Other features responsible for the pathophysiology of the SOP is altered signaling kisspeptinas contributing to the generation of pulses of GnRH, some studies have reported altered expression of Kiss1 and the number of neurons Kiss1 in the hypothalamus of various preclinical animal models SOP, generated by excessive androgen exposure, studies in rodent models SOP due to postnatal exposure to androgens have reported a persistent suppression of expression of hypothalamic Kiss1. Also they described other altered plants neuroendocrine pathways, which govern the function of $\mathrm{GnRH}$ neuron in preclinical models of SOP as a signaling impairment of gamma-aminobutyric acid (GABA) after an early androgenization in a model of SOP in mice.

As epigenetic several replication studies in Chinese and Caucasian subjects identified LH receptor choriogonadotropin (LHCGR) as a susceptibility gene for PCOS and hypomethylation of LHCGR cause hypersensitivity LH pulses, can by therefore be a plausible mechanism underlying susceptibility to PCOS, aromatase, encoded by CYP19A1, is another candidate gene, like estrogens are required for the selection and follicle growth, decreased aromatase can contribute to foliculogénesis defective.

Other mechanisms described in the literature, is altered activity of sympathetic nerves, alteration proposed in the sympathetic nerve activity to contribute to the aetiology of SOP as many common clinical symptoms of PCOS including central obesity, hyperinsulinemia and hyperandrogenaemia, are associated with a chronic increased activity of the sympathetic nervous system, the direct evaluation of sympathetic activity in women with PCOS revealed an additional association between sympathetic nerve activity and muscular PCOS independent of BMI, indirect markers of activity autonomous, including variability of heart rate and heart rate recovery after exercise,have shown that young women with PCOS show an increase of sympathetic and parasympathetic responses decreased, increased ovarian sympathetic tone in the SOP is based on the finding of a higher density of nerve fibers catecolaminérgicas in polycystic ovaries. ${ }^{8}$

\section{Diagnosis}

The polycystic ovary syndrome in adolescents is a persistent hyperandrogenic state oligoanovulatorio and menstrual abnormalities unexplained origin, so the diagnostic criteria for adolescents should be specific because if we use the criteria for adults traspolados the adolescent generate problematic, for it They describe Table 2 .

Table 2 Comparison of diagnostic criteria

\begin{tabular}{|c|c|}
\hline Diagnostic criteria for PCOS in adults & Diagnostic criteria for PCOS in adolescents \\
\hline $\begin{array}{l}\text { I. Phenotype I (SOP classical) clinical or biochemical } \\
\text { evidence of hiperandrogensimo, evidence oligo-ano- } \\
\text { vulation, polycystic ovaries evidence ultrasound }\end{array}$ & $\begin{array}{l}\text {-Anormalidad menstrual pattern } \\
\text { a. Abnormal for gynecological age } \\
\text { b. Persistence of symptoms for I or } 2 \text { years } \\
\text {-Evidence of hyperandrogenism }\end{array}$ \\
\hline $\begin{array}{l}\text { 2. Phenotype } 2 \text { clinical or biochemical evidence of } \\
\text { hiperandrogensimo, evidence oligo-anovulation }\end{array}$ & $\begin{array}{l}\text { a. Persistence of high levels of testosterone } \\
\text { b. Clinical moderate to severe hirsutism } \\
\text { c. Moderate to severe inflammatory acne, is an indication to } \\
\text { request profile androgens. }\end{array}$ \\
\hline
\end{tabular}


Table Continued...

\section{Diagnostic criteria for PCOS in adults \\ 3. Phenotype 3 (SOP ovulatory) clinical or bioche- mical evidence of hiperandrogensimo, polycystic ovarian morphology evidence ultrasound. \\ 4. Phenotype 4 (SOP not hyperandrogenic) evidence of oligo-anovulation, polycystic ovarian morphology evidence ultrasound.}

A. abnormal menstrual pattern

i. Abnormal for gynecological age

ii. Symptoms persist for 1 or 2 years

B. Evidence of hyperandrogenism

i. persistently elevated testosterone

ii. Clinical hyperandrogenism, ie, moderate to severe hirsutism, acne vulgaris moderate to severe inflammatory indicating request proof of androgens.

\section{Clinical manifestations}

\section{a. Ovary disorders}

Physiological anovulation in adolescents is a common phenomenon, the normal menstrual cyclicity adolescents differs only slightly from that of adults of reproductive age, cycles less than 19 days or more than 90 days are abnormal at any stage, $75 \%$ of cycles varies from 21 to 45 days during the first postmenarquico year and $95 \%$ of girls reach adult menstrual cycle of 21 to 40 days in the fifth gynecologic year, most of anovulation in adolescents is asymptomatic with cyclic menstrual bleeding that often occurs at intervals of 21 to 45 days even in the first year posmenarquico this paradox arises because the cyclical ovarian function immature usually occurs during these intervals. Most normal menstrual cycles are not ovulatory adolescents according to standard criteria have hormonal evidence luteal insufficiency, ie, serum hormonal changes during normal menstrual cycles adolescent confirm that the cyclic follicle is substantial but immature.

An abnormal menstrual bleeding pattern is almost always the Table 3 Types of menstrual dysfunction in adolescents

\section{Diagnostic criteria for PCOS in adolescents}

result of anovulatory cycles causing concern if persistent patterns of menstrual bleeding occur in 5\% of adolescents, one-third of patients with symptomatic anovulation teenager, which has rate overall longterm persistence, among girls evaluated by abnormal menstrual bleeding without clinical signs of hyperandrogenism, approximately half have elevated levels of androgen, the remeasurement of patients has shown that hyperandrogenaemia half of the resolved at approximately PCOS patients. Increase ovulation rates in the post-menarche reported percentages of $14 \%-22.9 \%, 25 \%-38 \%,-50 \% 44.8 \% 42.9 \%-48 \%$ and $63.2 \%-64 \%$ in children under 1 year, $1-2$ years, $2-3$ years, The study was planned in the early follicular phase and included patients with irregular cycles, urinary and serum sex steroid hormones were determined have been used as alternative methods for evaluating the presence of ovulation. In the early years of post-menarche, most healthy girls with irregular menstrual cycles are ovulating, this suggests that a history of irregular menstrual cycle should not be used as a clinical correlate of anovulation or oligoanovulación because healthy adult women with regular menstrual cycles have a prevalence of anovulation as $18 \%$ to $30 \%$, in addition it was reported that girls with regular menstrual cycles during late adolescence are anovulatory.

Menstrual dysfunction suggest abnormal levels of anovulation including less than six periods for one year during the second year postmenarquico, less than 9 periods per year during or after the fifth postmenarquico year or excessive menstrual bleeding, defined as a cycle that lasts more than 7 days or soaks a pad after one or two hours Table 3. PCOS patients the excessive uterine bleeding due to detachment of a hyperplastic endometrium exposed to insufficient progesterone, often called anovulatory uterine bleeding, increased estrogen secretion which cause a proliferative endometrium prolonged follicular phase, without But endometrial biopsy of these patients, revealed data compatible with secretory changes.

Types of abnormal uterine bleeding found in adolescent PCOS

\begin{tabular}{|c|c|}
\hline Description & Definition \\
\hline Primary amenorrhea & Lack of menarche at 15 years old or 3 years after the onset of breast development. \\
\hline \multirow[t]{3}{*}{ Secondary amenorrhea } & More than 90 days without a menstrual period, when already had menstruation \\
\hline & First posmenarquico year average cycle length of 90 days ( 4 periods / year) \\
\hline & Postmenarquico second year: average cycle length of 60 days ( 6 periods / year) \\
\hline & Postmenarquicos three to five years: average cycle length of 45 days ( 8 periods / year) \\
\hline & postmenarquicos sixth year cycle time $38-40$ days ( 9 periods / year) \\
\hline Excessive uterine bleeding & $\begin{array}{l}\text { Menstrual bleeding that occurs more frequently every |9-2| days or excessive (lasts } 7 \\
\text { days) or soaks I female pad or tampon every I-2 h }\end{array}$ \\
\hline
\end{tabular}

days) or soaks I female pad or tampon every I-2 $\mathrm{h}$ 
An abnormal menstrual pattern is almost always the result of cycles of anovulation, consequently, in the absence of an endocrine disorder, with abnormal menstrual pattern continues for one year, should be considered SOP, however, consensus warned PCOS in adolescents hyperandrogenic with menstrual abnormality for 2 years or more, so it is not recommended to diagnose PCOS, only provisional, that is, at risk of PCOS.9

By Radivojevic et al was reported, a study where the aim was to explore the post-menarche based on the development of the internal genitalia, ie, uterus, ovaries, 835 adolescents were studied, where I was classified according to their menstrual cycle regular and irregular the relationship between anthropometric parameters and sonographic findings of the pelvis were more studied, ovarian volume in the study were correlated with weight, BMI and body fat percentage of postmenarchal girls, no difference in uterine measures were among the groups studied, however, postmenarchal women with irregular cycles had higher ovarian volume.

Women with irregular cycles have a significantly higher than the adolescents who regularly ovulate average volume, this may be related to the possible development of SOP later in adulthood in girls with irregular cycles, implying that PCOS can occur at or shortly after puberty. ${ }^{10}$

\section{b. Clinical evaluation of hyperandrogenism}

Skin symptoms of hyperandrogenism are not necessarily present, however, the presence of moderate to severe hirsutism is evidence of clinical hyperandrogenism. Hirsutism is defined as an abnormal amount or increased hair dependent androgen areas and is greater than $5 \mathrm{~mm}$ long, in women is seen as a male distribution, ie, face, chest, back, abdomen, thighs and part upper arms, these areas are sensitive to androgens, thereby Ferriman and Gallwey conducted a study included 430 women between 15 and 74 years of age, however, most of them were older than 24 years, ie, 365430 Figure 1. the study did not specify the number of women aged 15 to 19 years time after menarche, a semiquantitative score for hirsutism assessing body hair in 11 sites developed: upper lip, chin, chest, upper back, lower back, upper abdomen, lower abdomen, arm, forearm, thigh and legs were classified from 0 to 4 , score 0 indicates absence of terminal hair and 01.04 increasing number of terminal hair progressively covers the entire area of the body, this study demonstrated an increase in hair growth with age and highlighted two areas forearm and legs were indifferent to hormonal hair growth in women aged 18 to 38 years old and, therefore, the initial score was modified to exclude the forearms and legs. The score may be affected by the use of a method of hair removal before evaluation.

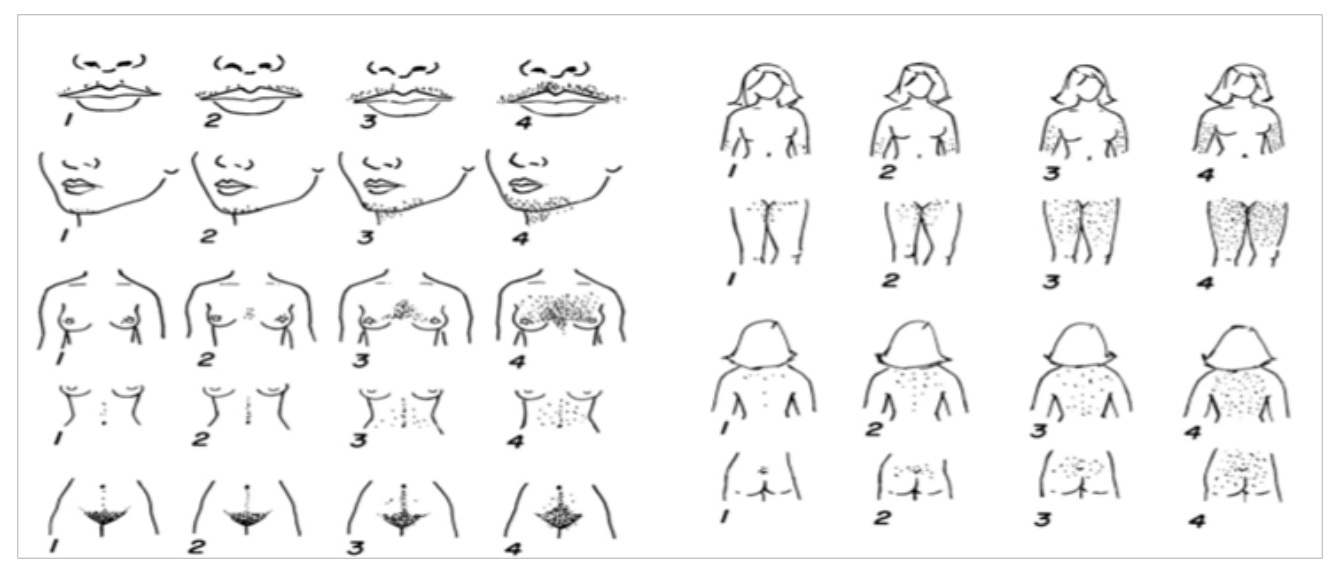

Figure I Index Ferriman-Gallwey (IFG).

The severity of hirsutism is qualified with a score of IFG: mild $<15$, 16-25 moderate and severe $>25$, with a total of 36 maximum score; hirsutism is a manifestation of hyperandrogenaemia for an answer in the polisebácea unit and its relationship to dihydrotestosterone, must be distinguished from hypertrichosis since the latter is the appearance of excessive hair with a non-androgen dependent distribution and where its cause is not attributed to an excess of circulating androgens.

\section{Acne}

The role of skin considered a steroidogenic tissue, research results confirm that it is a multifactorial actual inflammatory disease, where occurs alteration of the natural cycle of the pilosebaceous follicles which induces hyperseborrhoea, epithelial hyperproliferation in seboglandular duct and acroinfundíbulo, and the expression of proinflammatory cytokines with consequent formation of comedones and inflammatory lesions. Mild acne on the forehead and the center of the face is very common during the Adrenarche and adolescence, with an increasing prevalence of 15 to 20 years of age, the usual type of injury mild acne are comedonales injuries not are inflammatory and are generally low in number, that is, less than 20 lesions; moderate and severe acne is characterized by an increase in the number of comedonales and inflammatory lesions, severe acne can cause scarring and postinflammatory hyperpigmentation, is resistant to treatment and is less likely to occur during adolescence with a prevalence of up to $5 \%$ . Predictors of severe acne in adolescents include family history, race, early development of acne, increased body mass index, environmental factors, presence of menstrual abnormalities and higher levels of serum androgen, so the more moderate acne 10 injuries facial or severe over the years suggest premenarquicos hyperandrogenemia documented that the presence of inflammatory acne moderate to severe resistant topical treatment, antibiotic or orally, It is an indication for detection of hyperandrogenemia Table 4.

\section{Alopecia}

Patients with severe hyperandrogenism may have, alopecia although it is an unusual manifestation of hyperandrogenism, is defined as a diffuse thinning hair in the scalp area around the crown area may experience loss of bitemporal hair and hairline front, ie, 
male or female pattern christmas tree, ie, affects the crown widening the midline, is evaluated by Ludwig scale Figure 2.

Alternative manifestations of clinical or equivalent hirsutism hyperandrogenism include seborrhea, hyperhidrosis and suppurative hidradenitis, the latter is characterized by painful inflammatory nodules in intertriginous areas particularly in the underarm, frank virilization is unusual in SOP and should raise concern for other reasons hyperandrogenism. ${ }^{11}$

Table 4 System punctuation acne

\begin{tabular}{lll}
\hline \multicolumn{3}{l}{ Scoring system acne in adolescents } \\
\hline Severity & $\begin{array}{l}\text { Acne lesions } \\
\text { comedonal }\end{array}$ & Inflammatory acne lesions \\
\hline Mild & I-10 & I-10 \\
Moderate & II -25 & $1 \mathrm{I}-25$ \\
Severe & $>25$ & $>25$ \\
\hline
\end{tabular}

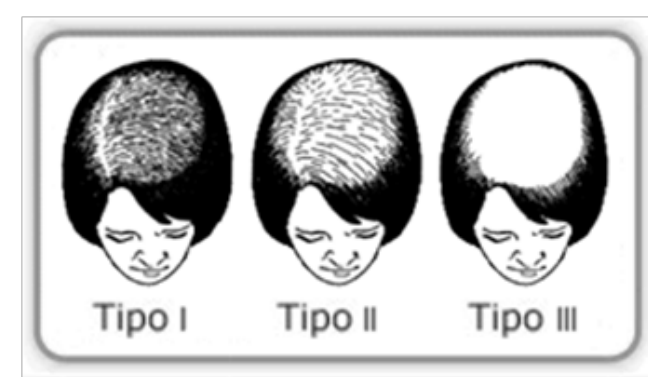

Figure 2 Scale ludwig.

\section{Assessment hyperandrogenaemia}

Documentation hyperandrogenemia requires reliable tests with normal well defined range, it is recommended to perform measurements of total testosterone and/or free, the latter elevated serum is the most sensitive indicator of hyperandrogenemia because the inactive portion of serum testosterone is free fraction. Serum SHBG govern the fraction of testosterone that is free, the routine measurement of more androgens free and total testosterone has not been documented, but can be considered androstenedione, dehydroepiandrosterone sulfate (DHEAS) and 17-hydroxyprogesterone the which is widely used to evaluate the adrenal hyperandrogenism, they are not routinely used in the evaluation of SOP, but are useful to exclude other conditions that can mimic the SOP. Although dihydrotestosterone generated in the average target tissue in most of the effects of testosterone, the serum level has little diagnostic value. However, accurate determinations of the concentrations of total and free testosterone are often problematic since the diurnal rhythm, the phase of the menstrual cycle and SHBG concentrations are biological variables influencing total testosterone.

Reliable analysis of free testosterone calculated concentration of free testosterone as the product of total testosterone and the fraction which is free of the binding of SHBG, combinations of SHBG and testosterone as the free androgen index or free testosterone calculated can help in the evaluation of hyperandrogenic states, the problem of calculating free testosterone a formula by Vermeulen was established in 1999 which corresponds to total / SHBG x 100 testosterone, the normal value is less than $5 \mathrm{nmol} / \mathrm{L}$. The criteria used to define hyperandrogenaemia adolescent girls are confused by development considerations, however, shortly after menarche, serum testosterone adolescent reaches adult levels.
Testosterone levels increase as anovulatory cycles of adolescents lengthen, therefore, limited data suggest that physiologic anovulation prolonged represents half of anovulatory cycles hiperandrogenémicos that resolve, biochemical evidence of hyperandrogenism, according indicated by the persistent elevation of serum total and/or free testosterone levels above the limit in adults, provide clear support for the presence of hyperandrogenism in an adolescent with PCOS symptoms in most laboratories, the upper limit approaches 55ng/dL for total testosterone and $9 \mathrm{pg} / \mathrm{ml}$ for free testosterone. Measuring AMH levels are increasingly used in adult women with PCOS. ${ }^{11}$

Göksever Celik et al conducted a study in which the study population were 276 women between 12 and 18 years, 469 patients between 22 and 28 years, with alteration in menstrual pattern, with or without evidence of ovarian morphology Polycystic by ultrasound, I demostrí levels of DHEA-S were higher in adolescents than in young adult women, the study also showed that prolactin levels were higher in adolescents than in young adult women, adolescents with menstrual irregularities had levels high androgen independent of the presence of polycystic ovary morphology. Therefore, it is proposed that the biochemical hyperandrogenism is the most important for the diagnosis of PCOS in this age group finding. ${ }^{12}$

Robert Rosenfield conducted a study, wherein the ratio of the biochemical ovarian function with the clinical spectrum observed in SOP and in asymptomatic patients with polycystic ovaries was evaluated, documenting that $95 \%$ of cases have a classic SOP, ie, phenotype 1 besides having a more severe hyperandrogenism and higher prevalence of polycystic ovarian morphology than other SOP, they have a significantly higher prevalence of glucose intolerance. There is a spectrum of ovarian androgen dysfunction ranging from subclinical to severe hyperandrogenaemia ovarian hyperandrogenism in most cases SOP classic. A minority of mild cases of SOP falls into this spectrum ovarian androgen dysfunction, but it seems that obesity is the basis of its hyperandrogenism or, less frequently. ${ }^{13}$

\section{Other manifestations of PCOS}

The insulin resistance reported in $30-70 \%$ of cases dysregulated involved in steroidogenesis and excess androgen production, hyperinsulinemic androgen excess is the most common cause of skin manifestations and participates in menstrual abnormalities pattern, vulnerability excess hyperinsulinemic androgens in adolescence is increased by restricting prenatal growth, this relationship may exist because early growth restriction reduces white adipogenesis and brown, myogenesis and other organogenesis, including hepatogenesis, involving modulations in circulating differentiation factor as preadipocyte factor 1 and follistatin,prenatal growth restriction is also associated with an increased risk of later development of insulin resistance and central adiposity.

The insulin resistance can be assessed by measuring levels of glucose and fasting insulin and the HOMA index with a cutoff of 2.5 , the definition of insulin resistance in adolescence, as well as the diagnostic value of different rates based on levels of fasting glucose and insulin, remains a subject of debate before an intervention can be justified oral tolerance test glucose hyperandrogenic girls with low birth weight, early menarche, acantosis nigricans and obesity, as these girls are at increased risk of glucose intolerance. The insulin resistance is exacerbated by obesity, present in $50-75 \%$ of obese adolescents and about $60 \%$ have metabolic syndrome, approximately $10 \%$ of women with PCOS have Type 2 Diabetes Mellitus before 40 years. The clinical manifestations of insulin resistance, is acanthosis 
nigricans, metabolic syndrome occurrence of risk factors for type 2 diabetes mellitus and cardiovascular disease, abdominal obesity, hyperglycemia, elevated triglycerides, low HDL cholesterol and hypertension, general metabolic syndrome has a prevalence of 3 times, breathing disorders, sleep apnea, described patients with PCOS as a latency longer sleep efficiency of the lowest sleep and a lower percentage of sleep and rapid eye movement REM which has been reflected in polysomnography disease, nonalcoholic fatty liver disease, its occurrence has been reported in 6-7\% of cases, demonstrated in a number of cases of obese patients. Evaluating the patient with PCOS should include, among others, the assessment of blood pressure, height and weight, waist index provide a clinical estimate of central adiposity, which is common among adolescents with excess androgens. ${ }^{14}$

Seda Ates et al in Turkey conducted a prospective study involving 77 adolescents aged 12-19 years, with oligo-anovulation and clinical hyperandrogenism, in order to determine the prevalence of metabolic syndrome in adolescents with PCOS. Adolescents with PCOS had a body mass index (BMI) significantly higher, waist circumference and LH levels, relative LH/FSH, triglycerides, insulin, HOMA-IR, free androgen index and lower levels of SHBG and FSH, had a higher prevalence of high BMI, HDL levels were lower, further noted that elevated androgen levels induce an unfavorable lipid profile, thereby conclude that BMI may be the main factor contributing to the development of metabolic abnormalities adolescents with PCOS. ${ }^{15}$

\section{Polycystic ovarian morphology ultrasound}

Criteria ovarian morphology poly cystic that have been used in adolescence are derived from adult criteria obtained from transvaginal ultrasound, which can not be performed on young teenagers as they are not sexually active, the transabdominal ultrasounds are not as reliable, especially girls are overweight or obese, currently representing at least one quarter of the population. The criteria for adults may not be appropriate during puberty because they are based on two parameters that can be commonly seen in healthy adolescent girls, one criterion is greater than $10 \mathrm{cc}$ ovarian volume, which is usually seen in adolescents whose ovarian volume increase after menarche. The other criterion is the number of follicles or ovarian cysts, ie, greater than 12 .

only $45 \%$ of patients with hyperandrogenism had an increase in the ovaries. The prevalence of ovarian enlargement in adolescents c hyperandrogenism was lower $35 \%$ of cases during the first 2 years after menarche and then about 50\% increase in ovarian size approached, in adults, is more common and can reach $80 \%$ to $85 \%$ in patients with severe forms of PCOS, so it is not considered useful in adolescent patients diagnosed with PCOS. ${ }^{16}$

The use of ultrasound as a diagnostic utility is controversial, since it has been reported as non-specific, associated with $40 \%$ of the adolescent population have changes in ovarian morphology, also the pelvic exam by transabdominal ultrasound results in lower resolution compared with endovaginal turn the prevalence of obesity makes it even more difficult and less useful to assess ovarian morphology therefore it has been proposed to replace the use of ultrasound for MRI and even the use of AMH, without But the most recent guidelines from the Endocrine society recommends the use of ovarian morphology in adolescents diagnostic criteria,therefore a cohort of adolescents with PCOS was performed and Rotterdam criteria modified were evaluated by transabdominal ultrasound, ie ovaries over 10 follicles and a volume of $10 \mathrm{cc}$, only $65 \%$ met sonographic criteria, $40 \%$ the number of follicles, $14 \%$ by volume and $46 \%$ number and ovarian volume, increasing age was not associated with reduction in follicles, the weight increase was associated with increased ovarian volume but not with the number of follicles, ultrasonographic changes were more prevalent in patients with PCOS, endometrial level, there was no relationship of endometrial line with polycystic ovarian morphology,therefore the incidence of sonographic changes using modified criteria Rotterdam was elevated in adolescents with PCOS so sonographic evaluation should be part of the diagnosis. ${ }^{17}$

\section{Utility MRI}

Moreover MRI as a diagnostic utility has shown preference for sensitivity and specificity in patients where transvaginal ultrasound is contraindicated, moreover transabdominal ultrasound despite studies that supported their use is still controversial and some authors report it as little optimal, however, have described studies in which MRI is used to examine ovarian morphology in PCOS, comparing their usefulness versus transvaginal and transabdominal ultrasound, Kenigsberg et al conducted a study where evaluated patients aged 1318 with PCO compared with an untreated control group SOP, pelvic MRI was performed with axial and coronal T2 and transvaginal and transabdominal ultrasound nubile patients,no clear diagnostic criteria for ovarian morphology were established using MRI so Rotterdam criteria were used cutoffs were performed, determining that ovarian volume is greater than or equal to $14 \mathrm{cc}$ and the number of larger follicles 17 , it is confirmed that PCOS patients have ovarian volume greater than teens without SOP and a greater number of follicles distributed peripherally, MRI allows better characterization of ovarian morphology polycystic compared to ultrasound, it was shown that ovarian volumes are smaller when measured by ultrasound, also do not allow individual follicles counts predominantly in obese patients, in contrast to the magnetic resonance distribution of follicles easily obtained, in turn detected that magnetic resonance $50 \%$ of patients already diagnosed with PCOS not meet the criteria of Rotterdam, therefore the usefulness of ultrasound seems be limited in the adolescent age group, the usefulness of imaging studies seems to have involvement in the confirmation of diagnosis or when dealing with an uncertain diagnosis, so its use should be reserved, ie, teenagers who meet diagnostic criteria SOP for clinical and laboratory findings may evaluation of ovarian morphology is not required, so most benefit to the use of MRI for diagnosis of PCOS is demonstrated, adding the calculated ovarian volume it is generated by computer based on the volumes in continuous axial segments, as opposed to the estimated volume by ultrasound based on the formula of a standard ellipse, however, although described, even lack of studies to reach consensus on ovarian morphology in adolescents with or without PCOS. ${ }^{18}$

\section{Differential diagnosis}

While SOP represents $80 \%$ of cases of androgen excess in postmenarchal women, however, there are other changes that occur as hyperandrogenism or disorder often have similar clinical findings and are difficult to distinguish from SOP.

i. nonclassical CAH ie late onset is the most common cause of excess androgens that occurs in adolescence, representing $4.2 \%$ of hyperandrogenic women world wide with a prevalence of $0.1-2 \%$, resulting deficit 21 -hydroxylase, patients have early puberty, hirsutism start in adolescence, anovulation and polycystic ovarian morphology, diagnosis is suggested by elevated serum levels of 17-hydroxyprogesterone. 
ii. classic $\mathrm{CAH}$, by 21-hydroxylase deficiency, diagnosed in childhood, presenting genital ambiguity and may be associated with a losing crisis salt, may develop during adolescence signs and symptoms of PCOS and clitoromegaly, ovarian morphology Polycystic can be the result of the direct effects of excess androgen or adrenal extraovarian remains in the ovaries, i.e, adrenal ectopic tissue

iii. Deficiency 3 beta hydroxysteroid dehydrogenase, rarely occurs in adolescence as a pattern SOP, classically presents in childhood with genital ambiguity due to adrenal virilizing congenital accompanied by symptoms of cortisol deficit aldosterone should be suspected when DHEAs be very high, the diagnosis is based on the determination of 17-hydroxypregnenolone greater than 10 standard deviations greater or $4500 \mathrm{ng} / \mathrm{dl}$.

iv. Deficit 11B hydroxylase classically presents with genital ambiguity due to congenital virilizing accompanied by hypertension, rarely less than $1 \%$ in adolescence as mild hyperandrogenism, hypertension and hypokalaemia, only 11 deoxycortisol responds to ACTH rising 5 times limit of normal.

v. Cushing syndrome rarely adrenal hyperplasia due to the appearance of hiperandrogenemica anovulation occurs.

vi. virilizing tumors, rare cause of severe hyperandrogenism, since more than half are malignant tumors, signs and symptoms are rapid onset, with symptoms virilising, hirsutism, alopecia, increased muscle mass, clitoromegaly without genital ambiguity.

vii. Hyperprolactinemia, the most common cause up to $95 \%$ of cases is the pituitary adenoma, however, may be present oligoanovulación and hyperandrogenism occurs in $40 \%$ of cases and $85 \%$ of patients have galactorrhea, the combination of hirsutism, galactorrhea and amenorrhea is called Forbes Albright syndrome probably hyperandrogenism results from the multiple effects of prolactin in androgen metabolism, evidence shows that hyperandrogenaemia is suppressible glucocorticoid treatment with dopamine agonists normalizes androgenic abnormalities.

viii. And acromegaly growth hormone excess increases levels IFG1 causing activity in multiple steroidogenic enzymes in the ovaries and adrenal gland.

ix. thyroid dysfunction interferes with the metabolism of gonadotropins, hypothyroidism also cause polycystic ovarian changes, also low levels of sex hormone binding globulin causes, can cause confusion with hirsutism because these patients have thickening hair.

x. Anabolic drugs cause virilization steroids and the amount of exogenous androgens causes polycystic ovaries, valproic acid increases transcription of CYP17 gene steroidogenic causing increase in serum testosterone.

\section{Increased cardiovascular risk and other comorbidities}

Early diagnosis of PCOS allows early control of the metabolic complications and the start of treatment that can mitigate potential complications associated with long term, the latter may be associated with hyperandrogenism and insulin resistance, leading to type 2 diabetes, hypertension, disease cardiovascular and dyslipidemia, fatty liver $\mathrm{n}$ alcoholic, endometrial cancer, increased risk of depression and anxiety, about 50 to $80 \%$ of adolescent PCOS patients are obese and 30 to $35 \%$ have insulin resistance, there by monitoring the insulin resistance is essential, tests glucose tolerance have a greater sensitivity and specificity than glycated hemoglobin,the American Diabetes Association recommended even in adolescents are over weight or obese with PCOS who are at least 10 years of age, ask glycated hemoglobin at diagnosis and after every 3 years.

PCOS patients are at increased risk of dyslipidemia, hypertension and atherosclerosis, described profile unfavorable lipid with increased LDL, decreased HDL and increased triglycerides, independent of BMI, therefore the association of SOP and cardiovascular effects demonstrates the importance of detecting blood pressure in adolescents with PCOS, documented elevated above 120 / 80 $\mathrm{mmHg}$, regarding NAFLD occurs at a rate five times higher than in patients without the diagnosis of PCOS, an approach reasonable to apply alanine aminotransferase at diagnosis particularly in patients with obesity and acanthosis nigricans, PCOS patients have a risk of $2.7 \%$ for endometrial cancer,due to anovulation and prolonged estrogen stimulation of the endometrium in turn are more prone to mood disorder, causing a negative effect on quality of life, patients with PCOS are often described as unattractive and less feminine, for this multidisciplinary management in this case with psychological health intervenes in preventing depression. ${ }^{19}$

It is recognized that metabolic syndrome and risk factors for cardiovascular disease are clearly increased in PCOS and cardiovascular health in general should be considered in a systematic review when comparing PCOS patients without PCOS risk of myocardial infarction and angina it increased in women with PCOS. As for all patients with PCOS should be offered regular monitoring of weight control, women who are over weight and obese women with PCOS, regardless of age, must have a lipid profile fasting, all women with PCOS should have a blood pressure measurement of each year, or more frequently depending on the overall risk of cardiovascular disease. $^{20}$

Previous studies have shown that adult women with PCOS have a smaller family size and fertility problems than healthy women, thus a cohort study was conducted from 1986 to explore the association of reproductive health, ie, diagnosis PCOS, infertility, pregnancy rates and abortions in early adulthood (26 years old) with a history of menstrual irregularity and high levels of androgens at age 16 , the current findings suggest that it is important to note that girls had menstrual irregularities at age 16 were more likely to suffer infertility problems at 26 not symptomatic girls, but did not show an increased risk of spontaneous abortions also showed a prevalence of $10.5 \%$ for patients with PCOS at age 26 did not differ BMI or weight, however, finds that the evidence hyperandrogenism and abnormalities in menstrual pattern during adolescence persist into adulthood, ${ }^{21}$ Moreover Robert Rosenfield conducted a similar study where the objective was to evaluate adolescent patients with PCOS and if this persists into adulthood during the study, a patient developed endometrial carcinoma at age 22 after being repeatedly rejected therapy cyclical progestin; this experience precautionary suggests that early diagnosis and counseling guidance on the prevention of endometrial hyperplasia should be important aspects of the management of PCOS adolescent, also tests Androgen ovarian function in hyperandrogenic adolescent it seems to be prognostic value since persistent SOP described during adulthood. ${ }^{22}$ 


\section{Treatment}

Treatment of adolescent patients with PCOS should ideally begin at an early stage and focus on both hyperandrogenism treatment and prevention and treatment of PCOS-related comorbidities such as obesity, insulin resistance and dyslipidemia.

Modification of lifestyle has been associated with improvements in endocrine and ovarian function in adult women with PCOS and is considered a first-line therapy in adolescents are overweight or obese, a study revealed that only the change of lifestyle was a $59 \%$ reduction in free androgen index with an increase of $122 \%$ in SHBG in adolescents aged 12 to 18 years old with a BMI $>95 \%$, are also 3.4 times more likely to have an improvement in menstrual function, the prevalence of metabolic syndrome decreases of $35 \%$ reductions in the homeostatic model assessment: insulin resistance (HOMA-IR), triglyceride and blood pressure. However the role of treatment should be multidisciplinary by the conditions described above.

\section{Oral contraceptives}

The combination of oral hormonal containing ethinylestradiol and progestogen suppresses ovarian androgen production, SHBG increases and decreases free testosterone concentrations. The estrogen component mainly leads to increased SHBG and subsequent decreased free androgen, while the progestin component protects against endometrial hyperplasia mediated unopposed estrogen, the progestin component mitigates the increased SHBG, so it is recommended choosing a progestogen with weak in the treatment of hyperandrogenemia androgenic activity, cyproterone acetate and drospirenone are two progestins with antiandrogenic activity but both are considered thrombogenic, desogestrel is a progestogens weak antiandrogenic activity.

\section{Insulin sensitizers}

The association between hyperinsulinemia and hyperandrogenemia in SOP is well recognized, the insulin sensitization induces ovulation and subsequent regular menses in non-obese adolescents with hyperandrogenism and history of premature puberty treated with 1275 mg metformin daily for 6 months. These patients also demonstrated improved scores hirsutism, insulin response and decreased serum androgen levels, a study of 18 teenage girls with PCOS treated with $1700 \mathrm{mg}$ a day of metformin for 6 months showed a reduction in BMI, decreased androgen levels and improvement in menstrual irregularity.

Thiazolidinediones are potent insulin sensitizers that have been effective in lean and obese adults with PCOS, with improved hyperandrogenemia, insulin resistance, anovulation, as well as decreasing inflammatory cytokines, a comparative study was conducted between drospirenone and ethinylestradiol versus rosiglitazone in obese adolescents with PCOS, rosiglitazone was associated with significant decreases in free testosterone and SHBG elevation, improved insulin sensitivity and hepatic and peripheral elevated levels of adiponectin. Furthermore, drospirenone and ethinylestradiol is associated with a worsening of lipoproteins LDL cholesterol and an increase in C-reactive protein, however pediatric use is not approved because of the risk of hepatotoxicity.

Inositol is a nutritional supplement that acts as a second messenger and has been shown to play a role in the transduction of insulin signaling, previous studies have focused on insulin resistance and hormonal profiles and gestational diabetes women with PCOS, however, one study found that while serum SHBG was favored with inositol, there were no statistically significant differences between inositol and placebo for BMI, waist-hip ratio, ovulation, serum testosterone, triglycerides, cholesterol, fasting glucose and fasting insulin, however, another study reported improvement in the rate of ovulation and menstrual cycles seem to improve with inositol in women with PCOS, therefore literature is limited,many key questions remain and research is prioritized.

\section{Androgen receptor blockers}

The spironolactone antiandrogen is commonly used as an adjunct therapy for treating hyperandrogenism, hirsutism specifically; spironolactone improves clinical hyperandrogenism and menstrual irregularity but no metabolic abnormalities, studies recommend the combination of metformin and spironolactone due to metabolic improvement, flutamide, will not be discussed because it is not approved for use in pediatric and concern and controversy persists over hepatotoxicity, cyproterone acetate, a steroidal progestogen with antiandrogenic activity, is commonly used in combination with ethinylestradiol in an oral contraceptive, as mentioned above..$^{20,23}$

\section{Treatment and follow-up in patients at risk of comorbidities}

tracking patients is suggested clinically every 3-6 months, lipid profile and glucose metabolism should be monitored, especially in patients who are over weight or obese, a curve of glucose tolerance is recommended every 3 to 5 years; more often if there is a significant weight gain or symptoms of type 2 diabetes For those patients who appear to be at risk for PCOS, but do not have biochemical hyperandrogenism, it is indicated close follow-up, serum androgens are recommended and, if clinically indicated, the detection curve tolerance glucose monitoring visits, also multidisciplinary treatment in patients with PCOS with a focus on reproduction and comprehensive approach to assessment and management of comorbidities as dysglycemia. ${ }^{24}$

\section{Discussion}

It is important to identify patients at risk for developing PCOS and establish early preventive measures; in patients who have developed SOP, establish a pharmacological treatment to reverse not only the symptoms, but to restore ovulatory function and delay or prevent the further development of co-morbidities. Although obesity, insulin resistance and hyperinsulinemia are common findings in adolescents with hyperandrogenism, these features should not be used to diagnose PCOS among adolescents, therefore, the diagnosis should be cautious and stick to the criteria according to literature revised, so you can provide the diagnosis at risk for PCOS. In adolescents in a provisional diagnosis of PCOS it was made.

\section{Conclusion}

The polycystic ovary syndrome in adolescents is a challenging diagnosis for the clinician, and there has been a consensus that persistent unexplained hyperandrogenic anovulation using age appropriate and stadium standards are appropriate criteria for diagnosis for PCOS in adolescents, warned by consensus not to label hyperandrogenic adolescents as SOP if the menstrual abnormality has not persisted for 2 years or more before that time, it is recommended to consider patients as at risk of PCOS and avoid misdiagnosis of changes at puberty, however, early diagnosis and early treatment change radically the prevalence of associated comorbidities, it 
should promote a holistic approach to maximize the future health of our patients, treatment point to a slow but steady return to a state of health with combination therapies that may vary over time and allow spontaneous ovulations, pregnancies without complications, decreased cardiovascular risk and a healthy reproductive life.

\section{Acknowledgments}

None.

\section{Funding}

None.

\section{Conflicts of interest}

Authors disclose no conflict of interests in publication of this study.

\section{References}

1. Rosenfield RL, Middleman AB, Geffner ME, et al. Definition clinical features and differential diagnosis of polycystic ovary syndrome in adolescents. UpToDate. 2019.

2. Rosenfield RL. The diagnosis of polycystic ovary in adolescence, Pediatrics. 2015;136(6):1154-1166.

3. Spritzer PM, Motta AB. Polycystic ovary syndrome and adolescence: Current concepts on diagnosis and treatment. $J$ Clin Pract Int. 2015;69(11):1236-1246.

4. Cirık DA, Dilbaz B. What do we know about prep metabolic syndrome in adolescents With PCOS? Turk J Gynecol Ger Assoc. 2014;15(1):49-55.

5. Rosenfield RL. Etiology and pathophysiology of polycystic ovary syndrome in adolescents. UpToDate. 2019.

6. Witchel SF, Roumimper H, Oberfield S. Polycystic ovary syndrome in adolescents. Endocrinol Metab Clin N Am. 2016;45(2):329-344.

7. Daghestani MH, Daghestani M, Daghistani M, et al. A study of ghrelin and leptin levels and Their relationship to metabolic profiles in obese and lean Saudi Women with polycystic ovary syndrome (PCOS). Lipids Health Dis. 2018;17(1):195.

8. Ibáñez L, Oberfield SE, Witchel S, et al. An international consortium update: Pathophysiology, diagnosis and treatment of polycystic ovary syndrome in adolescence. Horm Res Paediatr. 2017;88(6):371-395.

9. Pena AS, Doherty DA, Atkinson HC, et al. The majority of irregular menstrual cycles are ovulatory in adolescence: results of a prospective study. Arch Dis Child. 2018;103(3):235-239.

10. Radivojevic UD, Lazovic GB, Kravic-Stevovic TK, et al. Differences in anthropometric and ultrasonographic parameters between adolescent girls with regular and irregular menstrual cycles: a case study of 835 cases. $J$ Pediatr Adolesc Gynecol. 2014;27(4):227-231.
11. Pena AS, Metz M. What is adolescent polycystic ovary syndrome? $J$ Paediatr Child Health. 2018;54(4):351-355.

12. Çelik HG, Çelik E, Polat I. Evaluation of biochemical hyperandrogenism in adolescent girls menstrual with irregularities. Biochem $\mathrm{J}$ Med. 2018;37(1):7-11

13. Rosenfield RL. Polycystic ovary the polycystic ovary syndrome morphology-spectrum. J Pediatr Adolesc Gynecol. 2015;28(6):412-419.

14. Ibáñez L, Ong KK, López-Bermejo A, et al. Hyperinsulinaemic androgen excess in adolescent girls. Nat Rev Endocrinol. 2014;10(8):499-508.

15. Ates S, Aydin S, Ozcan P, et al. Clinical and metabolic characteristics of turkish polycystic ovary syndrome with adolescents. J Obstet Gynaecol. 2018;38(2):236-240.

16. Fruzzetti F, Campagna AM, Perini D, et al. Ovarian volume in Normal and hyperandrogenic adolescent women. Fertil Steril. 2015;104(1):196-199.

17. Youngster M, Ward VL, Blood EA, et al. Utility of ultrasound in the diagnosis of polycystic ovary syndrome in adolescents. Fertil Steril. 2014;102(5):1432-1438.

18. Kenigsberg LE, Agarwal C, Sin S, et al. Clinical utility of magnetic resonance imaging and ultrasonography for diagnosis of polycystic ovary syndrome in adolescent girls. Fertil Steril. 2015;104(5):1302-1309.

19. Fitzgerald S, DiVasta A, Gooding H. An update on PCOS in adolescents. Curr Opin Pediatr. 2018;30(4):459-465.

20. Teede H. International evidence-based guideline for the assessment and management of polycystic ovary syndrome 2018. Monash University on Behalf of the NHMRC, Center for Research Excellence in PCOS PCOS Alliance and the Australian; 2018.

21. West S, Lashen $\mathrm{H}$, Bloigu $\mathrm{A}$, et al. Irregular menstruation and hyperandrogenaemia in adolescence are associated With polycystic ovary syndrome and infertility in later life: Northern Finland 1986 birth cohort study. Hum Reprod. 2014;29(10):2339-2351.

22. Rosenfield RL, Ehrmann DA, Littlejohn EE. Polycystic ovary syndrome adolescent functional ovarian hyperandrogenism due to persists into adulthood. J Endocrinol Metab Clin. 2015;100(4):1537-1543.

23. Baldauff N, Arslanian S. Optimal management of polycystic ovary syndrome in adolescence. Arch Dis Child. 2015;100(11):1076-1083.

24. Sebastian MR, Wiemann CM, Bacha F, et al. Diagnostic evaluation, comorbidity screening, and treatment of polycystic ovary syndrome in adolescents in three specialty clinics. J Pediatr Adolesc Gynecol. 2018;31(4):367-371 